\title{
Kondo screening in $d$-wave superconductors in a Zeeman field and implications for STM spectra of Zn-doped cuprates
}

\author{
Matthias Vojta, Robert Zitzler, Ralf Bulla, and Thomas Pruschke \\ Theoretische Physik III, Elektronische Korrelationen und Magnetismus, \\ Universität Augsburg, 86135 Augsburg, Germany
}

(Dated: February 19, 2002)

\begin{abstract}
We consider the screening of an impurity moment in a $d$-wave superconductor under the influence of a Zeeman magnetic field. Using the Numerical Renormalization Group technique, we investigate the resulting pseudogap Kondo problem, in particular the field-induced crossover behavior in the vicinity of the zero-field boundary quantum phase transition. The impurity spectral function and the resulting changes in the local host density of states are calculated, giving specific predictions for high-field scanning tunneling microscopy measurements on impurity-doped cuprates.
\end{abstract}

\section{INTRODUCTION}

Impurity effects provide valuable information about bulk properties of correlated electronic systems such as cuprate superconductors. Remarkably, non-magnetic impurities like $\mathrm{Zn}$ and Li can induce a local momenton the neighboring lattice sites at intermediate energies 1 国 3 . On the other hand, $\mathrm{Zn}$ is known to act as pair breaker and strong scatterer, e.g. in low-temperature transport measurements, and at present it is not clear whether this behavior arises from bare potential scattering only, or if other effects like Kondo physics play a role.

Local impurity moments - arising either from magnetic impurities or induced by non-magnetic defects - interact with the low-energy bulk excitations. For cuprates at temperatures $T$ below the superconducting $T_{c}$, these are the fermionic quasiparticles of the $d$-wave superconductor and collective bosonic spin fluctuations. The coupling to the spin fluctuations mainly plays a role at intermediate energy scales of the order of the spin gap (an estimate is given by the position of the so-called resonance mode at $40 \mathrm{meV}$ ) - their effect on the impurity dynamics has been discussed recently in Ref. 6 .

For smaller energies, the dynamics of the impurity moments is governed by the interaction with the fermionic Bogoliubov quasiparticles. Importantly, their singleparticle density of states (DOS) vanishes at the Fermi energy, therefore the physics is quite distinct from the usual Kondo effect in metal Extensive studies of the

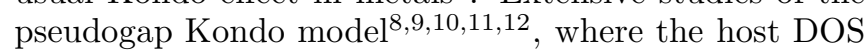
follows a power-law near the Fermi level, $\rho(\omega) \sim|\omega|^{r}$ ( $r=1$ for $d$-wave superconductors), have shown the existence of a boundary quantum phase transition between a free moment and a screened moment as the Kondo coupling is increased. Applied to the cuprates, we have argued 11 that this can lead to a strongly doping-dependent Kondo temperature, caused by the doping dependence of the size of the $d$-wave gap. These theoretical findings are consistent with NMR experiment 3 measuring the local susceptibility near a non-magnetic impurity. This susceptibility has been found to show Curie-Weiss-like behavior, with a Weiss temperature being finite at optimal doping but apparently vanishing for underdoped samples
- this implies the existence of unscreened moments in the underdoped regime down to temperatures of order $1 \mathrm{~K}$.

Interesting impurity signatures have also been observed in recent scanning tunneling microscopy (STM) experiments of the high temperature superconductor $\mathrm{Bi}_{2} \mathrm{Sr}_{2} \mathrm{CaCu}_{2} \mathrm{O}_{8+\delta}$ (BSCCO)13, 14 . Studies in the vicinity of $\mathrm{Zn}$ impurities on the $\mathrm{Cu}$ sites 3 have found a large peak in the differential conductance at small energies of 1-2 meV near the $\mathrm{Zn}$ site, with a characteristic spatial variation of the signal. This peak could represeptaquasibound state in a potential scattering model15.12.17, but such a state appears at low energies only for a range of larse potential values depending upenmicroscopic details17. Recently, it has been proposed 19, t1] that some of the properties of the $\mathrm{Zn}$ resonance can be explained by the Kondo spin dynamics of the magnetic moment induced by Zn. The screening of this moment by the Bogoliubov quasiparticles provides a natural low-energy scale (the Kondo temperature) explaining the energetic location of the peak seen in STM - in this case the potential scattering model might still apply as an effective model at low temperatures. However, not all features of the $\mathrm{Zn}$ resonance can be understood within the existing models, and important ingredients in the theoretical description, e.g., the actual tunneling path of the electrons from the tip to the $\mathrm{CuO}_{2}$ plane 18 , are not well known, calling for further experimental clarification.

In this paper we study the screening of an impurity moment embedded in a $d$-wave superconductor in a Zeeman magnetic field; we do neglect the changes in the quasiparticle spectrum due to the superflow induced by vortices which is appropriate for in-plane magnetic fields (parallel to the copper oxide layers) and for impurities located in between two vortices. In particular, we calculate the field-dependent impurity spectral density - based on these results, we propose that STM tunneling experiments in high magnetic fields will be able to distinguish between the possible sources of the observed impurity STM signal, and thus contribute to clarify the nature of impurity scattering in cuprate superconductors. 


\section{MODEL}

We begin by describing our model Hamiltonian, $H=$ $H_{\mathrm{BCS}}+H_{\mathrm{imp}}$, for a superconductor with a single impurity. The first term describes the host superconductor, which we model by a simple BCS Hamiltonian

$$
H_{\mathrm{BCS}}=\sum_{\mathbf{k}} \Psi_{\mathbf{k}}^{\dagger}\left[\left(\varepsilon_{\mathbf{k}}-\mu\right) \tau^{z}+h_{\mathrm{bulk}}+\Delta_{\mathbf{k}} \tau^{x}\right] \Psi_{\mathbf{k}} .
$$

Here $\Psi_{\mathbf{k}}=\left(c_{\mathbf{k} \uparrow}, c_{-\mathbf{k} \downarrow}^{\dagger}\right)$ is a Nambu spinor at momentum $\mathbf{k}=\left(k_{x}, k_{y}\right)$ in standard notation. $\tau^{x, y, z}$ are Pauli matrices in particle-hole space, and $\mu$ is the chemical potential. For the kinetic energy, $\varepsilon_{\mathbf{k}}$, we have first $(t)$, second $\left(t^{\prime}\right)$, and third $\left(t^{\prime \prime}\right)$ neighbor hopping, while we assume a $d$-wave form for the BCS gap function $\Delta_{\mathbf{k}}=$ $\left(\Delta_{0} / 2\right)\left(\cos k_{x}-\cos k_{y}\right)$. The spin quantization axis lies in the direction of the (in-plane) Zeeman field $h_{\text {bulk }}$.

The Green's function of the conduction electrons described by $H_{\mathrm{BCS}}$ is $G^{0}(\mathbf{k}, \omega)=\left[\omega-\left(\varepsilon_{k}-\mu\right) \tau^{z}-h_{\text {bulk }}-\right.$ $\left.\Delta_{k} \tau^{x}\right]^{-1}$ in matrix notation. The influence of a Zeeman magnetic field on a $d$-wave BCS superconductor has been studied in Ref. 19. For low temperatures and small fields, $h_{\text {bulk }} \ll \Delta_{0}$, the field-induced change in the gap function $\Delta_{\mathbf{k}}$ can be neglected. Thus the main effect of the Zeeman field is to split the quasiparticle spectrum by $\pm h_{\text {bulk }}$, which leads to a finite DOS at the Fermi level.

We will not consider the coupling of the external field to the orbital motion of the electrons - the primary effect of the field-induced superflow would be an additional shift in the quasiparticle spectrum. Neglecting this Doppler effect is approximately justified for fields applied parallel to the $\mathrm{CuO}_{2}$ planes of the system: Due to the layered crystal structure and strong anisotropy, the orbital effect of the magnetic field is much weaker for in-plane fields, and vortex effects are likely to be small19. Furthermore, we expect our approximation to apply also for fields in $c$-axis direction, i.e., perpendicular to the planes, for impurities located in between two vortices where the superflow tends to cancel and hence the Doppler shift is small.

The impurity, located at $\mathbf{r}_{0}=(0,0)$, is described by an effective model consisting of a scattering potential $U$ and a Kondo term for the spin-1/2 impurity spin $\mathbf{S}$ :

$$
H_{\mathrm{imp}}=\sum_{\mathbf{R} \in \mathcal{N}} J_{\mathbf{R}} \mathbf{S} \cdot \mathbf{s}_{\mathbf{R}}+S^{z} h_{\mathrm{loc}}+U c_{\alpha}^{\dagger}\left(\mathbf{r}_{0}\right) c_{\alpha}\left(\mathbf{r}_{0}\right) .
$$

where $\mathbf{s}_{\mathbf{R}}=N_{s}^{-1} \sum_{\mathbf{k k}^{\prime} \alpha \beta} \mathrm{e}^{\mathrm{i}\left(\mathbf{k}-\mathbf{k}^{\prime}\right) \mathbf{R}} c_{\mathbf{k} \alpha}^{\dagger} \frac{1}{2} \sigma_{\alpha \beta} c_{\mathbf{k}^{\prime} \beta}$ is the conduction band spin operator at site $\mathbf{R}$. As discussed in Refs. 6. 10, 11 we will consider two cases: (i) a local magnetic moment where $\mathcal{N}=\left\{\mathbf{r}_{0}\right\}$, and (ii) a spatially extended magnetic moment (modelling $\mathrm{Zn}$ ) where $\mathcal{N}$ is a set of sites in the neighborhood of $\mathbf{r}_{0}$; guided by NMR experiment $\mathbb{A}$ we take $\mathcal{N}$ to be the four neighbors of $\mathbf{r}_{0}$, i.e., $J_{\mathbf{R}}=J$ for $\mathbf{R}-\mathbf{r}_{0}=( \pm 1,0),(0, \pm 1)$ and $J_{\mathbf{R}}=0$ otherwise. Note that this four-site model describes an induced moment fluctuating as a single entity, in agreement a)

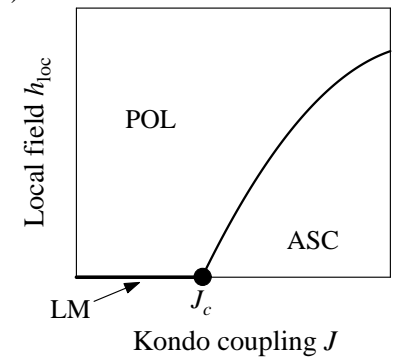

b)

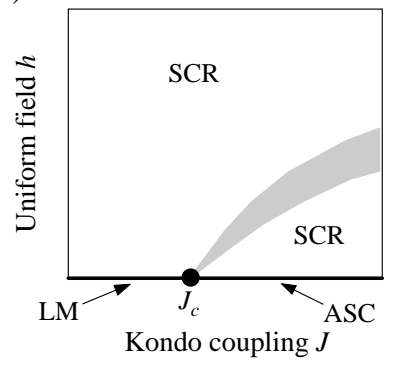

FIG. 1: Schematic zero-temperature phase diagrams for the asymmetric pseudogap Kondo model in a Zeeman field, deduced from NRG. a) Local field $h_{\text {loc }}$ only, i.e., for $h_{\text {bulk }}=0$. There is a line of continuous phase transitions at finite fields, terminating in the multicritical point at $J=J_{c}, h_{\mathrm{loc}}=0$. (The phases LM, ASC, and POL are explained in the text.) b) Uniform field $h=h_{\text {loc }}=h_{\text {bulk }}$. Here, the asymptotic low$T$, low-frequency behavior at finite fields is always that of a conventional Kondo-screened spin in a magnetic field (SCR phase), but there is a sharp crossover separating regions which are dominated by ASC and POL behavior at finite energies.

with spin relaxation experiments 0 . The Kondo term in the four-site case defines a multichannel Kondo problem; however, the low-energy physics in the present situation is dominated by a single channel given by the $d$-wavelike linear combination of the conduction electrons on the four neighboring sites 10.11 .

The potential scattering term in (2) can be accounted for exactly:

$$
\begin{aligned}
& G\left(\mathbf{r}, \mathbf{r}^{\prime}, \omega\right)=G^{0}\left(\mathbf{r}-\mathbf{r}^{\prime}, \omega\right)+U G^{0}\left(\mathbf{r}-\mathbf{r}_{0}, \omega\right) \\
& \times \tau^{z}\left[1-U G^{0}((0,0), \omega) \tau^{z}\right]^{-1} G^{0}\left(\mathbf{r}_{0}-\mathbf{r}^{\prime}, \omega\right) .
\end{aligned}
$$

The Kondo problem we consider in the following is then defined by the spin-dependent DOS seen by the impurity. For a single-site impurity we have

$$
\rho_{\text {eff }, \alpha}(\omega)=-\operatorname{Im} G_{\alpha \alpha}\left(\mathbf{r}_{0}, \mathbf{r}_{0}, \operatorname{sgn}(\alpha) \omega\right) / \pi
$$

with $\rho_{\text {eff, } \alpha}(\omega) \sim|\omega|$ at zero field, whereas the extended four-site impurity leads to

$$
\rho_{\text {eff }, \alpha}(\omega)=-\frac{1}{4 \pi} \operatorname{Im} \sum_{\mathbf{R}, \mathbf{R}^{\prime}} \varphi_{\mathbf{R}} \varphi_{\mathbf{R}^{\prime}} G_{\alpha \alpha}\left(\mathbf{R}, \mathbf{R}^{\prime}, \operatorname{sgn}(\alpha) \omega\right)
$$

which behaves as $\rho_{\mathrm{eff}, \alpha}(\omega) \sim|\omega|^{3}$ at $h_{\text {bulk }}=0$. Here, $\varphi_{\mathbf{R}}=+[-] 1$ for $\mathbf{R}-\mathbf{r}_{0}=( \pm 1,0)[(0, \pm 1)]$ describe the $d$ wave channel of the four-site impurity, $G$ is the $\Psi$ Green's function (3) in Nambu notation, and $\operatorname{sgn}(\alpha)= \pm 1$ for $\alpha=\uparrow, \downarrow$.

\section{BOUNDARY CRITICAL POINTS AND CROSSOVER BEHAVIOR}

In the following, we describe our numerical results for the above Kondo model in an external field, obtained 

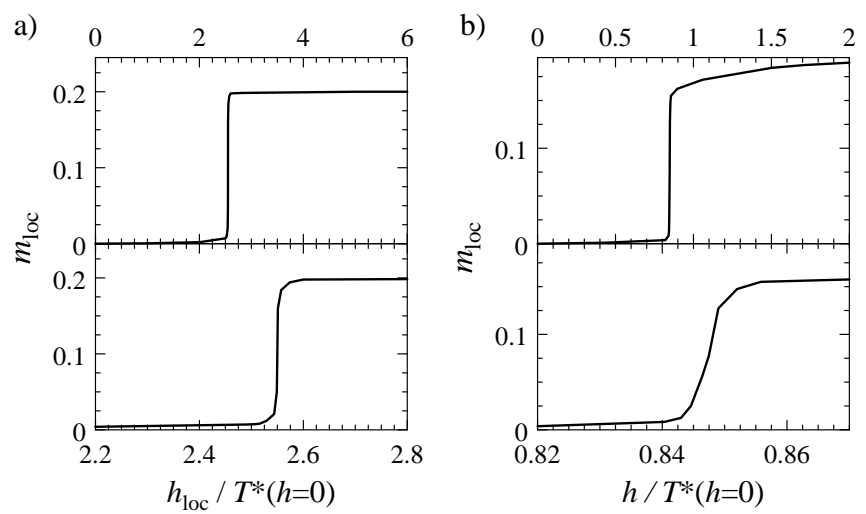

FIG. 2: NRG results for the local impurity magnetization $m_{\text {loc }}=\left\langle S^{z}\right\rangle$, calculated for a four-site impurity in a cuprate band structure $\left(t=-0.15 \mathrm{eV}, t^{\prime}=-t / 4, t^{\prime \prime}=t / 12 \Delta_{0}=\right.$ $0.04 \mathrm{eV}, \mu=-0.14 \mathrm{eV}$ ) with $J_{c} \approx 0.23 \mathrm{eV}$ for $U=022$, as a function of the applied field. a) Local field $h_{\text {loc }}$ only, for a $J>$ $J_{c}$ with $T^{*} \approx 5 \mathrm{~K}$. At the phase transition the magnetization follows a power law, $\left|m_{\mathrm{loc}}-m_{\mathrm{loc}, c}\right| \sim\left|h_{\mathrm{loc}}-h_{\mathrm{loc}, c}\right|^{\zeta}$, with $\zeta \approx 0.1$. b) Uniform field $h=h_{\text {loc }}=h_{\text {bulk }}$, for a $J>J_{c}$ with $T^{*} \approx 25 \mathrm{~K}$. Here, the magnetization evolves smoothly through the crossover region of Fig. Ifb. The lower figures show zooms of the upper ones.

with Wilson's Numerical Renormalization Group (NRG) technique20. The zero-field pseudogap Kondo problem in the presence of particle-hole asymmetry is known to show a boundary quantum phase transition between an asymmetric strong-coupling (ASC) phase with a screened impurity moment and a local moment (LM) phase with Curie behavior of the impurity susceptibility 11 .

We start by considering the case of a local field only, $h_{\text {loc }} \neq 0$ but $h_{\text {bulk }}=0$, such that the host DOS is unaffected by the field. From the analysis of the fixed points of the NRG calculation we have found that even in a finite local field there is still a phase transition upon varying the Kondo coupling. The $T=0$ phase diagram deduced from NRG is schematically shown in Fig. 1 $1 \mathrm{a}$ - there is a line of finite-field transitions ending in the zero-field transition point known from earlier work 911 which can be viewed as a multicritical point. The three stable phases are easily understood: The ASC phase at $J>J_{c}$ extends to finite field (with weak spin polarization for $h_{\text {loc }} \neq 0$ ), whereas the LM phase at $J<J_{c}$ with a free impurity spin exists at zero field only. Applying a field at $J<J_{c}$ immediately leads to a phase with strong polarization of the impurity spin, therefore we term this phase POL.

All transitions found are continuous phase transitions, characterized by a small energy scale $T^{*}$, which vanishes at the transition, and defines the crossover energy above which quantum-critical behavior is observed. In the ASC phase at zero field, $T^{*}(J, h=0)$ can be identified with the Kondo temperature 11 , i.e., with the binding energy of the Kondo singlet. The transition from ASC to POL is characterized by breaking this singlet by the external field consequently the critical field $h_{\mathrm{loc}, c}$ close to the multi- critical point is given by $T^{*}(J, h=0)$ times a prefactor depending on the DOS exponent $r$. Note that this novel field-induced Kondo transition is unique to the pseudogap model studied here - in the metallic host case $(r=0)$ the application of a field leads only to a gradual suppression of the Kondo effect 21 .

From the above discussion we expect that a uniform Zeeman field $h_{\text {bulk }}=h_{\text {loc }}=h$ removes the critical point, because a finite $h_{\text {bulk }}$ leads to a finite DOS at the Fermi level. Our numerical calculations show that this is indeed the case - for any finite $h$ the asymptotic zero-temperature behavior is that of a conventional (i.e. metallic-host) Kondo-screened spin in a magnetic field. However, the characteristic energy scale below which this behavior occurs is tiny, as it is exponentially small in the field-induced DOS at the Fermi level. (The same applies to effects arising from the small low-energy DOS induced by a finite confentration of impurities, i.e., the so-called impurity band11.) Therefore, at practically accessible temperatures the finite-field behavior is determined by the physics of the POL and ASC phases described above, and both regimes are now separated by a sharp crossover, Fig. 1 b. This crossover field turns out to be somewhat smaller than the critical local field in Fig. 17a; their ratio depends on the field-induced DOS at the Fermi level (i.e. the DOS exponent $r$ ).

The properties of the phases are reflected in the fieldinduced impurity magnetization, Fig. 2. This quantity is finite for any non-zero field, but shows a power-law singularity at the transition in the local-field case (Fig. 2a). Note that the behavior of $m_{\text {loc }}$ near the transition is not universal but depends on $J$ - the reason is that the finitefield transitions actually represent a line of critical fixed points in the RG sense. In the uniform-field case, Fig. 2b, the singularity in $m_{\text {loc }}$ is replaced by a relatively sharp crossover.

\section{IMPURITY $T$ MATRIX AND STM}

Turning to the dynamic properties of the Kondo impurity, we now discuss the conduction electron $T$ matrix, $T(\omega)$, which is the important input quantity for the calculation of the local DOS measured by STM. The $T$ matrix spectral density, $\rho_{T_{\alpha}}(\omega)=-\operatorname{Im} T_{\alpha}(\omega) / \pi$, in zero external field has been studied in Ref. 11 using NRG. It was found that $\rho_{T}(\omega) \sim|\omega|^{r}$ in the stable LM and ASC phases. The critical fipint at $J_{c}$ is characterized by $\rho_{T}(\omega) \sim|\omega|^{-r}$ (for $r \leq 1)^{11}$.12. The crossover from quantum-critical $|\omega|^{-r}$ behavior at high energies to $|\omega|^{r}$ behavior at low energies leads to a characteristic peak in the spectral density on one (!) side of the Fermi level at an energy $T^{*}(J, h=0)$, which can be viewed as Kondo peak (note that the problem at hand is intrinsically particle-hole asymmetric).

For finite field, the spin degeneracy is lifted: applying a small field to the system with $J>J_{c}$ splits the Kondo peak, in a manner roughly similar to the field-induced peak splitting in the conventional (metallic) Kondo ef- 


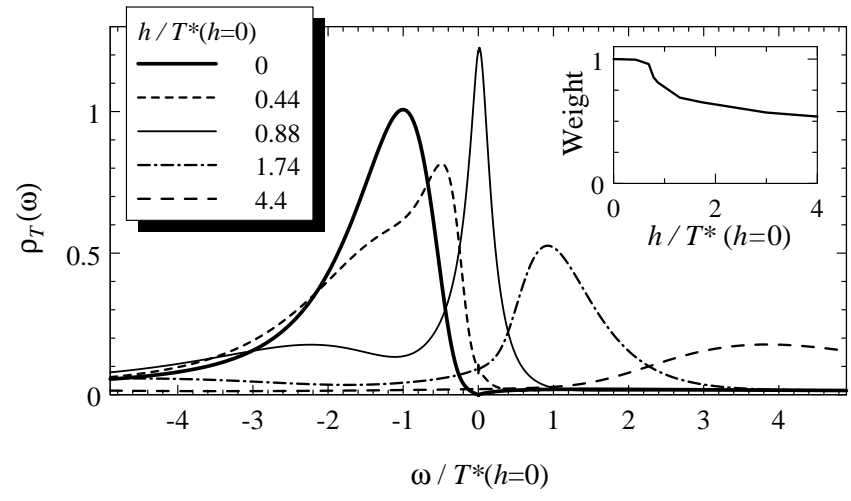

FIG. 3: Typical NRG results for the $T$ matrix spectral density, $\sum_{\alpha} \rho_{T_{\alpha}}(\omega)$, at fixed $J>J_{\alpha}$ for different uniform fields $h$, calculated for a local impurity 23 . The height of the Kondo peak reaches its maximum in the crossover region of Fig. $1 \mathrm{~b}$ and rapidly decreases for higher fields. Inset: Normalized weight of the Kondo peak, integrated from $\omega=-10 T^{*}$ to $10 T^{*}$.

fect 21. For $h_{\text {bulk }}=0$ the low-frequency limit of $\rho_{T}(\omega)$ still behaves as $|\omega|^{r}$; of course, finite $h_{\text {bulk }}$ will lead to a finite value of $\rho_{T}(\omega=0)$. The simple peak-splitting picture is modified when the field becomes comparable to the zero-field peak energy, i.e., close to the transition/crossover lines in Fig. 11. In the case of a purely local field, we observe that the spectral peak for one spin direction approaches the Fermi level and becomes sharper as $\left|h_{\text {loc }}-h_{\text {loc }, c}\right|$ is decreased; it diverges at the phase transition as $|\omega|^{-r}$, whereas the spectrum for the opposite spin broadens and remains non-critical. For a uniform field, this effect is again smeared out: in the crossover region of Fig. 11 b the spectral function shows a peak with a finite, small width at the Fermi level, see Fig. 3. Remarkably, the weight of the Kondo peak (inset of Fig. 3) stays nearly constant at small fields (ASC regime), but rapidly decreases (POL regime) once the crossover region is passed.

Applying a field to a system with $J<J_{c}$ immediately suppresses the spectral function for one spin species whereas the other one shows a smaller peak which moves away from the Fermi level and gradually looses intensity - so there are no drastic effects as suggested by the phase diagram.

Using the $T$ matrix from the NRG method 23 it is straightformard to determine the local conduction electron DOS 11 as measured by STM. Fig. 1 shows a set of calculated on-site tunneling spectra for a four-site impurity in a cuprate band structure, which is supposed to describe the moment induced by a Zn impurity in BSCCO. For a zero-field peak position of $-1.4 \mathrm{meV}$, the crossover region of Fig. 17b is reached at an in-plane field of approximately $20 \mathrm{~T}$. For larger fields, the peak moves to positive bias and looses intensity similar to the inset of Fig. 3. The spatial intensity pattern of the signal is nearly field-independent; it is alternating from site to site, i.e.,

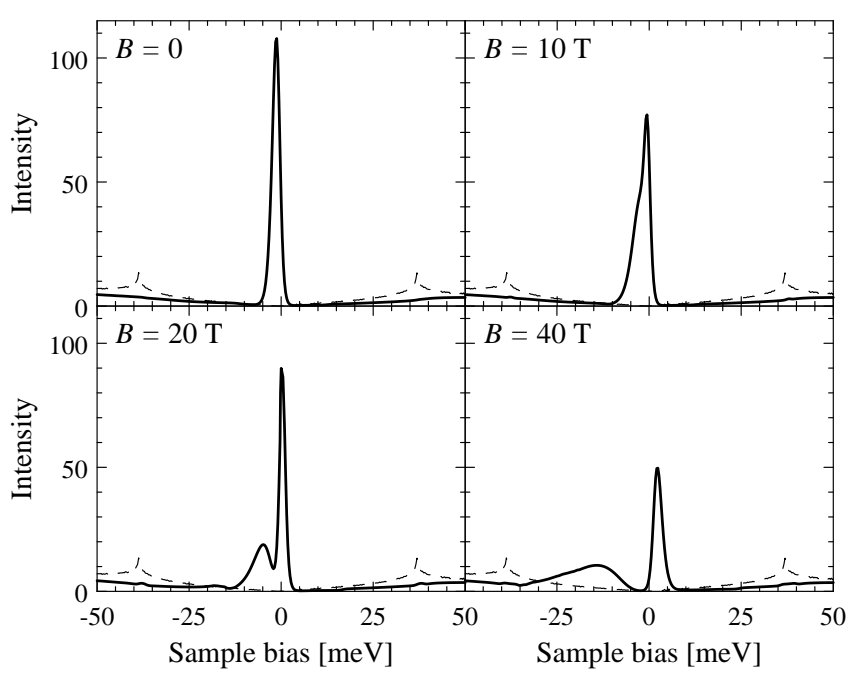

FIG. 4: Field evolution of the tunneling DOS for the foursite Kondo impurity, measured on the imprity site, without taking into account a possible filter effect 18 . The host paramaters are as in Fig. 2, the Kondo coupling is chosen to yield a zero-field peak at $-1.4 \mathrm{meV}\left(T^{*}=15 \mathrm{~K}\right)$, the potential scattering is $U=0$. The results have been broadened corresponding to a measurement temperature of $4.5 \mathrm{~K}$; the dashed line shows the bulk DOS. (The external field $B$ is related to $h$ by $h=g \mu_{B} B / 2$.)

the nearest neighbor of the impurity has a very small intensity yhereas the second neighbor has again higher intensity 10 , 21 . 5 . Fig. [ 4 shows results for zero potential scattering $U$, we note that no qualitative modifications occur for small and moderate $U$ values up to the hopping energy $t$, see also Ref. 11.

\section{CONCLUSIONS}

Summarizing, we have studied the effect of a Zeeman field on the Kondo screening of impurity moments embedded in a host with a pseudogap density of states. The application of a local magnetic field leads to a novel field-induced boundary phase transition, associated with breaking up the Kondo singlet - this transition has characteristic signatures in the local magnetization and the impurity spectral function. In the context of $\mathrm{Zn}$ impurities in cuprate $d$-wave superconductors we predict a characteristic evolution of the impurity STM spectrum with applied field which can be tested in future experiments. The spectra in Fig. A can be contrasted to the results for a pure potential scattering model24, where the scattering peak is simply split by the Zeeman energy, but is not modified in its total weight - therefore these experiments will help to clarify the general role played by non-magnetic impurities in cuprates. 


\section{Acknowledgments}

ported by the DFG through SFB 484 .

We thank S. Davis, D. Morr, A. Polkovnikov, and S. Sachdev for valuable discussions. This research was sup-

1 H. Alloul, P. Mendels, H. Casalta, J.-F. Marucco, and J. Arabski, Phys. Rev. Lett. 67, 3140 (1991).

2 D. L. Sisson, S. G. Doettinger, A. Kapitulnik, R. Liang, D. A. Bonn, and W. N. Hardy, Phys. Rev. B 61, 3604 (2000).

3 J. Bobroff, W. A. MacFarlane, H. Alloul, P. Mendels, N. Blanchard, G. Collin, and J.-F. Marucco, Phys. Rev. Lett. 83, 4381 (1999); J. Bobroff, H. Alloul, W. A. MacFarlane, P. Mendels, N. Blanchard, G. Collin, and J.F. Marucco, Phys. Rev. Lett. 86, 4116 (2001).

4 M.-H. Julien, T. Feher, M. Horvatic, C. Berthier O. N. Bakharev, P. Segransan, G. Collin, and J.F. Marucco, Phys. Rev. Lett. 84, 3422 (2000).

5 W. A. MacFarlane, J. Bobroff, H. Alloul, P. Mendels, N. Blanchard, G. Collin, and J.-F. Marucco, Phys. Rev. Lett. 85, 1108 (2000).

6 S. Sachdev, C. Buragohain, and M. Vojta, Science 286, 2479 (1999); M. Vojta, C. Buragohain, and S. Sachdev, Phys. Rev. B 61, 15152 (2000).

7 A. C. Hewson, The Kondo Problem to Heavy Fermions, Cambridge University Press, Cambridge (1997).

8 D. Withoff and E. Fradkin, Phys. Rev. Lett. 64, 1835 (1990); L. S. Borkowski and P. J. Hirschfeld, Phys. Rev. B 46, 9274 (1992); C. R. Cassanello and E. Fradkin, Phys. Rev. B 53, 15079 (1996) and 56, 11246 (1997).

9 K. Ingersent, Phys. Rev. B 54, 11936 (1996); C. GonzalezBuxton and K. Ingersent, Phys. Rev. B 57, 14254 (1998); K. Ingersent and Q. Si, Phys. Rev. Lett. 89, 076403 (2002).

10 A. Polkovnikov, S. Sachdev, and M. Vojta, Phys. Rev. Lett. 86, 296 (2001).

11 M. Vojta and R. Bulla, Phys. Rev. B 65, 014511 (2002).

12 M. Vojta, Phys. Rev. Lett. 87, 097202 (2001).

13 E. W. Hudson, S. H. Pan, A. K. Gupta, K. W. Ng, and
J. C. Davis, Science 285, 88 (1999); S. H. Pan, E. W. Hudson, K. M. Lang, H. Eisaki, S. Uchida, and J. C. Davis, Nature 403, 746 (2000).

14 E. W. Hudson, K. M. Lang, V. Madhavan, S. H. Pan, H. Eisaki, S. Uchida, and J. C. Davis, Nature 411, 920 (2001).

15 A. V. Balatsky, M. I. Salkola, and A. Rosengren, Phys. Rev. B 51, 15547 (1995); M. I. Salkola, A. V. Balatsky, and D. J. Scalapino, Phys. Rev. Lett. 77, 1841 (1996).

16 H. Tsuchiura, Y. Tanaka, M. Ogata, and S. Kashiwaya, J. Phys. Soc. Jpn. 68, 2510 (1999).

17 W. A. Atkinson, P. J. Hirschfeld, A. H. MacDonald, and K. Ziegler, Phys. Rev. Lett. 85, 3926 (2000).

18 J. X. Zhu, C. S. Ting, and C. R. Hu, Phys. Rev. B 62, 6027 (2000); I. Martin, A. V. Balatsky, and J. Zaanen, Phys. Rev. Lett. 88, 097003 (2002).

19 K. Yang and S. L. Sondhi, Phys. Rev. B 57, 8566 (1998).

${ }^{20}$ K. G. Wilson, Rev. Mod. Phys. 47, 773 (1975).

21 T. A. Costi, Phys. Rev. Lett. 85, 1504 (2000), W. Hofstetter, Phys. Rev. Lett. 85, 1508 (2000).

22 This corrects a numerical error in our earlier computation, Ref. 11.

23 Technically, the calculation of the Kondo impurity $T$ matrix within NRG is done using an equivalent singleimpurity Anderson model in the Kondo limit. To obtain accurate results at finite energies, the impurity spectral function has been calculated via the impurity self energy, as described in: R. Bulla, A. C. Hewson, and T. Pruschke, J. Phys.: Condens. Matter 10, 8365 (1998).

24 C. Grimaldi, Phys. Rev. B 65, 094502 (2002). 